\title{
Double-quantum NMR spectroscopy based on finite pulse RFDR
}

\author{
Yao-Hung Tseng ${ }^{\mathrm{a}, \mathrm{b}}$, Yun Mou ${ }^{\mathrm{a}}$, Chung-Yuan Mou ${ }^{\mathrm{a}, \mathrm{b}}$, Jerry C.C. Chan ${ }^{\mathrm{a}, *}$ \\ ${ }^{a}$ Department of Chemistry, National Taiwan University, College of Science, No. 1, Section 4, Roosevelt Road, Taipei 10617, Taiwan \\ ${ }^{\mathrm{b}}$ Center of Condensed Matter, National Taiwan University, No. 1, Section 4, Roosevelt Road, Taipei, Taiwan
}

Received 21 October 2004; received in revised form 21 January 2005

\begin{abstract}
We demonstrate that the finite pulse RFDR sequence (J. Chem. Phys. 114 (2001) 8473) can be used effectively for ${ }^{31} \mathrm{P}$ doublequantum NMR spectroscopy at a spinning frequency of $10 \mathrm{kHz}$. The ${ }^{31} \mathrm{P}$ NMR data measured for hydroxyapatite and octacalcium phosphate show that sizable double-quantum excitation efficiency can be obtained with the ratio of the recoupling field to spinning frequency set equal to 1.67 .
\end{abstract}

(C) 2005 Elsevier Inc. All rights reserved.

Keywords: MAS; P-31 NMR; Double-quantum; DQ; fpRFDR

\section{Introduction}

Double-quantum (DQ) NMR spectroscopy under magic angle spinning has been the major research area in the solid-state NMR community for many years. Since the pioneering work of Tycko [1], numerous pulse sequences designed for DQ NMR have been reported in the literature [2-5]. In particular, the symmetry theory developed by Levitt, Nielsen and co-workers has led to the development of a splendid collection of pulse sequences suitable for DQ NMR spectroscopy under fast magic-angle spinning [6-11]. However, the implementation of these windowless pulse sequences is not trivial if phase transient problem is severe. Sometimes a damping resistor has to be added in parallel to the sample coil in order to reduce the detrimental effect of phase glitches [12]. The down side of adding a damping resistor is that the probe sensitivity would then be deteriorated. On the other hand, the RFDR sequence $[13,14]$ or the like have the advantage that all the pulses are well separated and each of the pulses is of constant amplitude and rf phase. Hence, the phase glitch problem

\footnotetext{
${ }^{*}$ Corresponding author. Fax: + 886223636359.

E-mail address: chanjcc@ntu.edu.tw (J.C.C. Chan).
}

becomes immaterial after regular spectrometer tune-up procedure. Although RFDR is usually used for zeroquantum NMR, Blanco and Tycko demonstrated that the RFDR sequence can be used for DQ excitation after being flanked by a pair of $\pi / 2$ pulses [15].

Recently, Ishii reported that the finite pulse effect on the regular RFDR sequence can be exploited to provide dipolar recoupling under fast spinning condition [16]. In contrast to the conventional RFDR pulse sequence, the lowest-order average Hamiltonian of this so-called fpRFDR sequence has the same operator form of a static dipole-dipole Hamiltonian except for a scaling factor. Hence it is possible to combine fpRFDR with many elegant pulse sequences developed for static solids $[17,18]$. In the context of DQ excitation, it is rather straightforward to incorporate fpRFDR into the DQ excitation sequence proposed by Blanco and Tycko. Accordingly, the ratio between the $\pi$ pulse length and the rotor period is simply adjusted to 0.3 [16]. This simple approach was first illustrated in the high-speed magic-angle spinning multiple quantum (HSMAS-MQ) experiment by Oyler and Tycko, in which they were focusing on ${ }^{13} \mathrm{C}$ systems [18]. In the subsequent discussion, we will evaluate this approach with respect to ${ }^{31} \mathrm{P}$ DQ NMR by numerical simulations and 
experimental measurements on octacalcium phosphate and hydroxyapatite. For convenience, we refer this approach to as HSMAS-DQ in the subsequent discussion.

\section{Experimental}

\subsection{Sample preparation}

Octacalcium phosphate was prepared and characterized as described previously [19]. Hydroxyapatite was used as received (Sigma-Aldrich).

\subsection{Numerical simulations}

All numerical studies in this work were carried out using SIMPSON (version 1.1.0) [20]. For our simulations, the maximum time step over which the Hamiltonian is approximated to be time-independent was set to $3.0 \mu$ s. Typically, a powder averaging scheme containing 100 REPULSION angles $(\alpha$ and $\beta$ ) [21] and $18 \gamma$ angles was chosen. Relaxation effects were ignored. Two series of spin clusters used for our simulations were constructed based on the structural parameters of octacalcium phosphate [22] and hydroxyapatite [23]. For octacalcium phosphate there are six non-equivalent sites for phosphorus, where $\mathrm{P} 1-\mathrm{P} 4$ are $\mathrm{PO}_{4}^{3-}$ groups while $\mathrm{P} 5$ and $\mathrm{P} 6$ are $\mathrm{HPO}_{4}^{2-}$ groups. For hydroxyapatite there is only one phosphorous site $\left(\mathrm{PO}_{4}^{3-}\right)$. In the octacalcium phosphate series, we have constructed seven spin clusters, viz. 2-P, 4-P, 5-P, 7-P, 2P-H, 4P-H and $5 \mathrm{P}-2 \mathrm{H}$. As shown in Fig. 1(a), the two-spin system (2$\mathrm{P})$ involves $\mathrm{P} 3$ and $\mathrm{P} 6$. The 4-P, 5-P and 7-P spin clusters are constructed by adding two, three and five of the nearest phosphorous neighbors, respectively, to the basic 2-P system. When the acidic protons of the P5 and $\mathrm{P} 6$ species $\left(\mathrm{HPO}_{4}^{2-}\right)$ were added to the 2-P, 4-P and
5-P clusters, we obtained the $2 \mathrm{P}-\mathrm{H}, 4 \mathrm{P}-\mathrm{H}$ and $5 \mathrm{P}-2 \mathrm{H}$ clusters, respectively. In the hydroxyapatite series, similarly we have constructed three different spin clusters, viz. 2-P, 3-P and 5-P (Fig. 1b). For both series the chemical shift spans of $\mathrm{PO}_{4}^{3-}(40 \mathrm{ppm})$ and $\mathrm{HPO}_{4}^{2-}$ $(121 \mathrm{ppm})$ were taken from the experimental data measured for hydroxyapatite and brushite, respectively [24]. For simplicity the orientations of the CSA tensors with respect to the dipolar framework were set arbitrarily.

\subsection{Solid-state NMR}

All NMR experiments were carried out at ${ }^{31} \mathrm{P}$ and ${ }^{1} \mathrm{H}$ frequencies of 121.5 and $300.1 \mathrm{MHz}$, respectively, on a Bruker DSX300 NMR spectrometer equipped with a commercial $4 \mathrm{~mm}$ MAS-NMR probe. All spectra were measured at a spinning frequency of $10 \mathrm{kHz}$. MAS frequency variation was limited to $\pm 3 \mathrm{~Hz}$ using a commercial pneumatic control unit. The sample was confined to the middle $1 / 3$ of the rotor volume using Teflon spacers. Chemical shifts were externally referenced to $85 \%$ phosphoric acid. The pulse sequence used for the ${ }^{31} \mathrm{P}$ DQ measurements is shown in Fig. 2. To prepare the initial spin system identically for each transient, a saturation comb was applied prior to the recycle delay. Recycle delay was set to $8 \mathrm{~s}$. During the DQ excitation and reconversion periods, the ${ }^{31} \mathrm{P} \pi / 2$ and $\pi$ pulses were set to 5 and $30 \mu$ s long, respectively. The $\pi$ pulse trains were phase cycled according to the XY-16 scheme [25]. The DQ excitation and reconversion times were both set to $3.2 \mathrm{~ms}$ unless stated otherwise. The tachometer signals for pulse sequence synchronization were filtered with a home-built phase-locked loop circuit. Proton decoupling was set to $85 \mathrm{kHz}$. A $z$-filter of $30 \mathrm{~ms}$ without proton decoupling was applied after the reconversion period. The standard four-step phase cycling of the excitation block $(0, \pi / 2, \pi, 3 \pi / 2)$ and
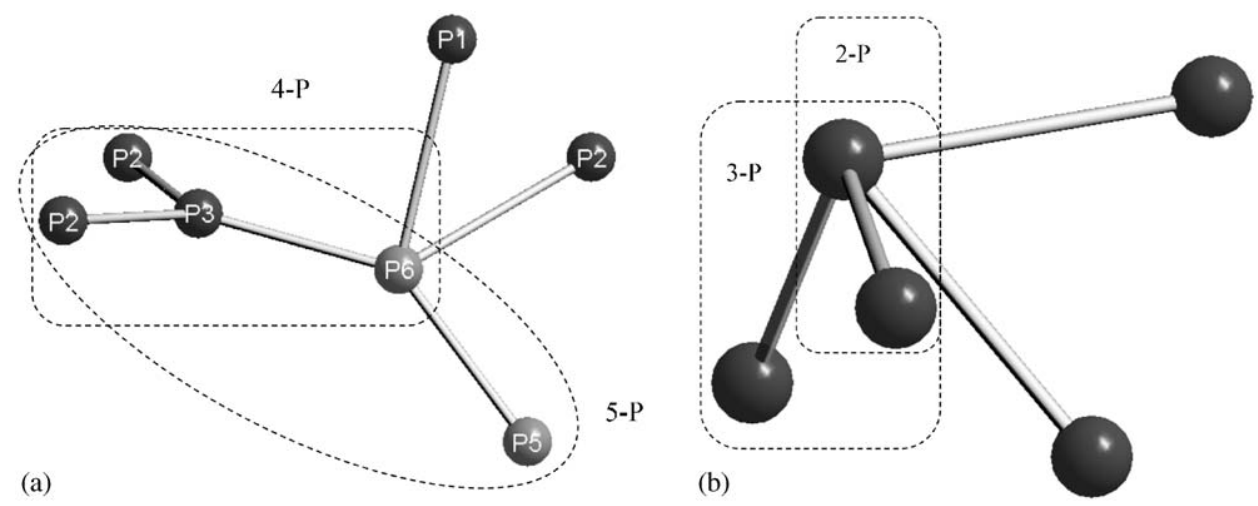

Fig. 1. The spin clusters constructed for numerical simulations. (a) Octacalcium phosphate series: 2-P, P3 and P6; 4-P, spins enclosed by the rectangle; 5-P, spins enclosed by the ellipse; 7-P, all spins. The dark and gray spheres represent $\mathrm{PO}_{4}^{3-}$ and $\mathrm{HPO}_{4}^{3-}$, respectively. (b) Hydroxyapatite series: 2-P, 3-P, 5-P. 


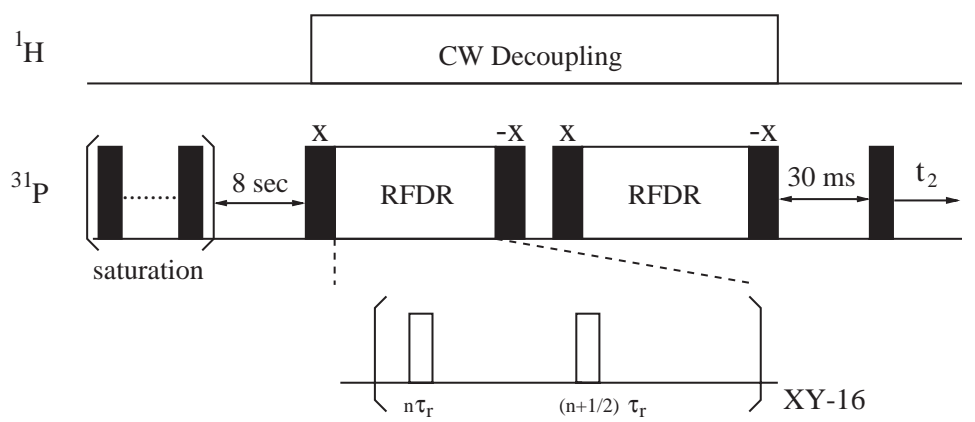

Fig. 2. Pulse sequences used for the ${ }^{31} \mathrm{P}$ DQ experiment. The filled and open rectangles denotes $\pi / 2$ and $\pi$ pulses, respectively.

receiver phase $(0, \pi, 0, \pi)$ was used to select the DQ coherence [26]. Probe ringdown and dc offset artifacts were suppressed by inverting the phase of the first $\pi / 2$ pulse and alternating addition and subtraction of FID signals. Together with CYCLOPS, our phase cycling has a total of 32 steps. For the two-dimensional DQ spectrum measurement, quadrature detection in the $F_{1}$ dimension was achieved by the hypercomplex approach. For each $t_{1}$ increment 32 transients were accumulated, and a total of 48 increments were done at steps of two rotor periods.

\section{Results}

\subsection{Octacalcium phosphate}

\subsubsection{Numerical simulations}

To evaluate the utility of HSMAS-DQ, we have carried out a series of simulations on the spin clusters 2$\mathrm{P}, 4-\mathrm{P}, 5-\mathrm{P}$ and 7-P (octacalcium phosphate series). The closest internuclear distances of selected phosphorus spin pairs are listed in Table 1. Other simulation parameters were set equal to the experimental settings. To evaluate the effect of proton decoupling on the DQ excitation efficiency, we focus on a ${ }^{31} \mathrm{P}-{ }^{31} \mathrm{P}$ spin pair formed by $\mathrm{PO}_{4}^{3-}$ and $\mathrm{HPO}_{4}^{2-}$ in close proximity, i.e. P3-P6. Fig. 3(a) shows the numerical results of the excitation efficiency of the P3-P6 DQ coherence in different spin clusters. There is a dramatic decrease in the excitation efficiency when the cluster size increases from two spins to four spins. This observation can be understood by the fact that the passive spins in close proximity provide a strong dipolar dephasing of the excited DQ coherence. As expected, such dephasing effects are comparable for the 4-P, 5-P and 7-P systems. Note that P4 is not included in our spin clusters because it is rather remote from P3 or P6 (Table 1). To investigate the effect of proton decoupling, we repeat the above calculations on the $2 \mathrm{P}-\mathrm{H}, 4 \mathrm{P}-\mathrm{H}$ and $5 \mathrm{P}-2 \mathrm{H}$ spin clusters using a proton decoupling field of $85 \mathrm{kHz}$ (Fig. 3b). Because the proton decoupling field is much
Table 1

Closest internuclear distances (in $\AA$ ) of selected phosphorus spin pairs in octacalcium phosphate

\begin{tabular}{llll}
\hline Auto-correlation & \multicolumn{3}{c}{ Cross-correlation } \\
\hline P1-P1 & 4.043 & P2-P3 & 4.017 \\
P4-P4 & 4.041 & P3-P6 & 4.206 \\
P5-P5 & 4.042 & P5-P6 & 4.350 \\
P2-P2 & 6.835 & P1-P4 & 4.697 \\
P3-P3 & 6.835 & P1-P2 & 4.707 \\
P6-P6 & 6.835 & P2-P4 & 4.722 \\
& & P2-P6 & 4.951 \\
& & P1-P6 & 4.969 \\
& & P4-P3 & 5.052 \\
& & P1-P3 & 5.494 \\
& & P4-P6 & 5.986 \\
\hline
\end{tabular}

The data are grouped to facilitate the analysis of the two-dimensional ${ }^{31} \mathrm{P}$ DQ spectrum (Fig. 4).

larger than the ${ }^{31} \mathrm{P}$ recoupling field, the excitation profiles shown in Figs. 3a and b are quite similar.

\subsubsection{Two-dimensional $D Q$ spectrum}

The two-dimensional ${ }^{31} \mathrm{P}$ DQ spectrum of octacalcium phosphate is shown in Fig. 4(a), in which there are eight sets of auto- and cross-correlation peaks. For convenience, in Fig. 4(b) we also reproduce the corresponding one-dimensional ${ }^{31} \mathrm{P}$ MAS spectrum and the spectral assignments reported earlier [19]. The correlation peaks of P2-P6, P1-P6, P1-P3 and P5-P5/ $\mathrm{P} 5-\mathrm{P} 6$ were not observed in our previous work, where we employed windowless pulse sequence at a spinning frequency of $6 \mathrm{kHz}$ [19]. Although the ${ }^{31} \mathrm{P}$ chemical shift span of $\mathrm{HPO}_{4}^{2-}$ is about $121 \mathrm{ppm}$ [24], the relatively high intensity of the P5-P5/P5-P6 correlation peak demonstrates that the DQ excitation efficiency is not significantly attenuated by large CSA effect. Altogether these results illustrate the advantages of the HSMAS-DQ approach that the experiment can be carried out at fast spinning frequency and that the ratio of the recoupling to decoupling fields are small. In view of the fact that the closest distance between P1 and P3 is $5.494 \AA$, it is 

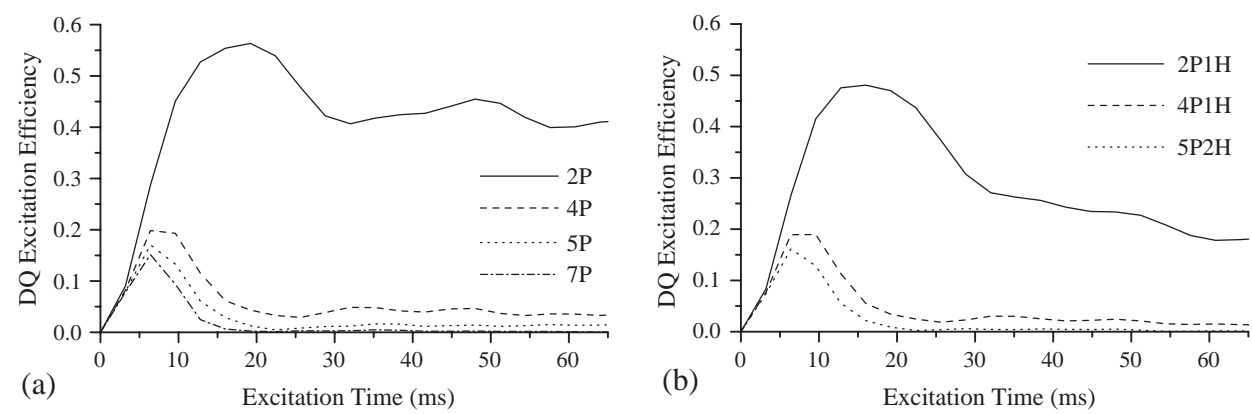

Fig. 3. Numerical simulations of the excitation efficiency of the P3-P6 DQ coherence in different spin clusters (octacalcium phosphate series). (a) 2$\mathrm{P}$, 4-P, 5-P and 7-P. (b) $2 \mathrm{P}-1 \mathrm{H}, 4 \mathrm{P}-1 \mathrm{H}$ and $5 \mathrm{P}-2 \mathrm{H}$.
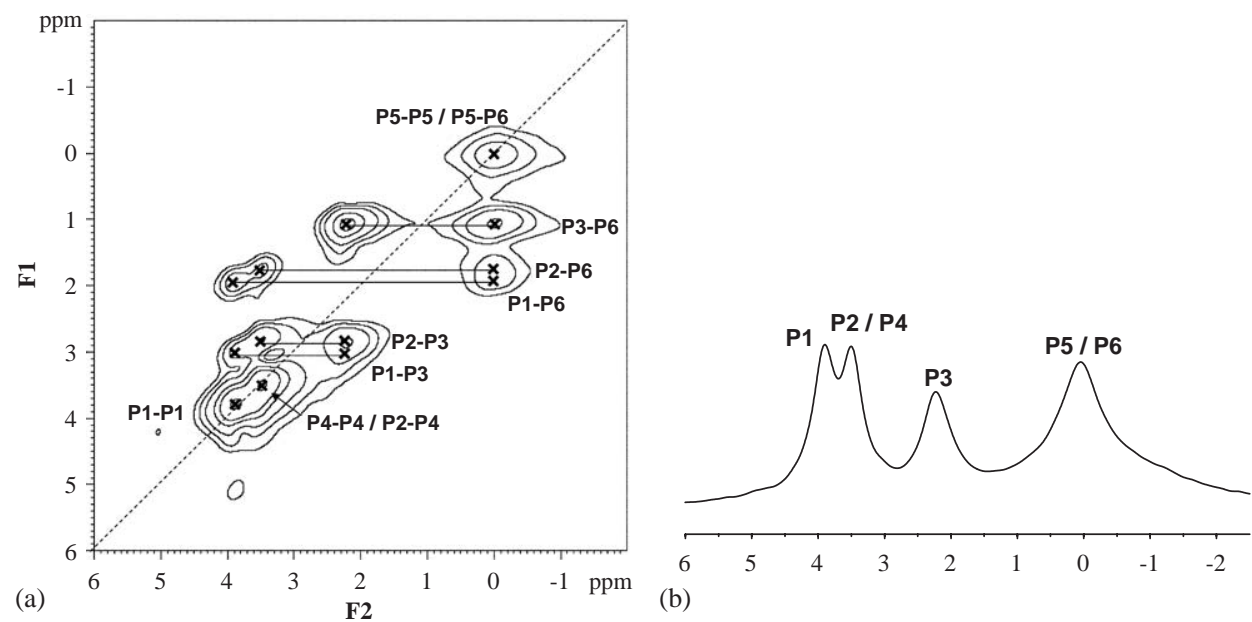

(b)

Fig. 4. NMR spectra of octacalcium phosphate measured under $10 \mathrm{kHz}$ spinning frequency: (a) Two-dimensional ${ }^{31} \mathrm{P}$ DQ based on the HSMAS-DQ sequence; (b) MAS.

surprising that the intensity of the $\mathrm{P} 1-\mathrm{P} 3$ cross-correlation peak is comparable to that of P2-P3 (4.017 $\mathrm{A}$ ). This observation cannot be explained by different spectral assignments. We speculate that the $\mathrm{P} 1-\mathrm{P} 3$ cross-correlation arises from a relayed coherence transfer $\mathrm{P} 1 \leftrightarrow \mathrm{P} 6 \leftrightarrow \mathrm{P} 3$ because both $\mathrm{P} 1-\mathrm{P} 6$ and $\mathrm{P} 6-\mathrm{P} 3$ are in close proximity. To minimize the duty cycle in the proton channel, the $z$-filter was applied without proton decoupling. Therefore, higher order spin diffusion may also occur during the $z$-filtering period [27].

The ${ }^{31} \mathrm{P}$ Bloch decay signal after the saturation comb and relaxation delay (same as the DQ experiment) was taken as a reference signal. After normalizing the P3-P6 cross-peak intensities by the geometric mean of $\mathrm{P} 3$ and P6 reference signal intensities, the excitation efficiency for the P3-P6 DQ coherence is estimated to be ca. $20 \%$.

\subsection{Hydroxyapatite}

Because hydroxyapatite has one phosphorous site only, it is a good model compound for the study of DQ coherence excitation profile. The closest ${ }^{31} \mathrm{P}-{ }^{31} \mathrm{P}$ distance in hydroxyapatite is $4.115 \AA$. The numerical simulations and the experimental data were shown in Fig. 5(a). Similar to what has been observed in octacalcium phosphate, the maximum DQ coherence excitation efficiency decreases as the spin cluster size increases. Nevertheless, the extent of the diminishment is not as dramatic as what we observed for octacalcium phosphate. The reason is simply due to the fact that all the spins in the cluster will contribute (different combinations of two spins) to the DQ coherence. As expected the simulation results converge towards the experimental data as the cluster size increases. Since we do not include any relaxation parameters in our simulations, the agreement between the simulation and the experimental data becomes worse at longer excitation times.

To compare the RFDR-based DQ excitation strategy [15] and the HSMAS-DQ approach, we repeat our simulations and experimental measurements by shortening all the $\pi$ pulses from 30 to $7 \mu \mathrm{s}$. The results shown in Fig. 5(b) indicate that all the excitation profile maxima invariably appear at longer excitation times than those in Fig. 5(a). This observation is consistent with the simulation results of Oyler and Tycko [18]. 

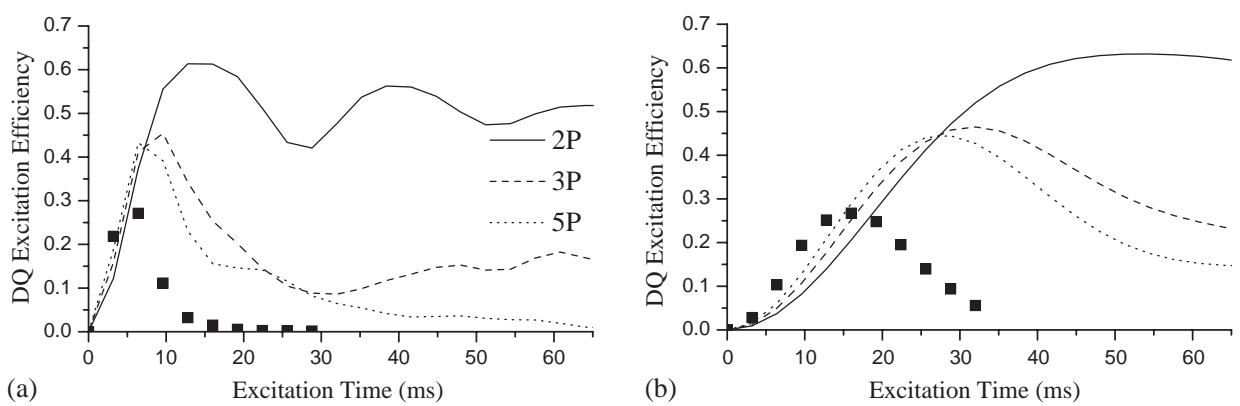

Fig. 5. Numerical simulations and experimental measurements of the excitation profile of the ${ }^{31} \mathrm{P} D Q$ coherence in hydroxyapatite at a spinning frequency of $10 \mathrm{kHz}$. The symbols, representing the experimental data, are larger than the error bars. (a) fpRFDR, $\pi$ pulse $=30 \mu \mathrm{s}$ (b) RFDR, $\pi$ pulse $=7 \mu$ s.

Overall, both numerical and experimental data illustrate that the HSMAS-DQ method is a favorable option for ${ }^{31} \mathrm{P}$ DQ NMR under a spinning frequency of $10 \mathrm{kHz}$.

\section{Discussion}

Our simulation results illustrate that the excitation of DQ coherence is inherently difficult in multiple-spin system due to the dephasing effect of other passive spins. The strong dependence of the excitation profile on the spin cluster size is in line with the conclusion that distance measurement by DQ NMR spectroscopy in homonuclear spin system is practical only if the two-spin approximation holds [28].

In this work, we have demonstrated that HSMAS-DQ can be used effectively for ${ }^{31} \mathrm{P}$ DQ NMR spectroscopy. In spite of the favorable experimental results shown here, we make no claim that the HSMAS-DQ approach is in general superior to the $\mathrm{C} / \mathrm{R}$ or any other windowless pulse sequences [8]. In fact, the theoretical maximum DQ excitation efficiency of HSMAS-DQ is less than those $\gamma$-encoded pulse sequences [6]. Nevertheless, we do recommend the HSMAS-DQ approach originally developed in Tycko group when the phase transient problem is a serious one.

\section{Acknowledgment}

This work was supported by grants from the National Science Council and the Ministry of Education. We thank Professor John Balbach (Physics Department, George Washington University) and Professor Carl Michal (Physics Department, University of British Columbia) for sharing with us their circuit designs for tachometer signal clean up. We thank Dr. Hans Foerster (Bruker, Karlsruhe) for technical advice. We thank one of the anonymous reviewers for bringing Ref. [27] to our attention.

\section{References}

[1] R. Tycko, G. Dabbagh, Chem. Phys. Lett. 173 (1990) 461-465.

[2] R. Tycko, Annu. Rev. Phys. Chem. 52 (2001) 575-606.

[3] M. Baldus, Prog. Nucl. Magn. Reson. Spectrosc. 41 (2002) 1-47.

[4] S. Luca, H. Heise, M. Baldus, Acc. Chem. Res. 36 (2003) 858-865.

[5] I. Schnell, Prog. Nucl. Magn. Reson. Spectrosc. 45 (2004) 145-207.

[6] Y.K. Lee, N.D. Kurur, M. Helmle, O.G. Johannessen, N.C. Nielsen, M.H. Levitt, Chem. Phys. Lett. 242 (1995) 304-309.

[7] M. Carravetta, M. Eden, X. Zhao, A. Brinkmann, M.H. Levitt, Chem. Phys. Lett. 321 (2000) 205-215.

[8] M.H. Levitt, Symmetry-Based Pulse Sequence in Magic-Angle Spinning Solid-State NMR, Wiley, Chichester, 2002.

[9] M. Hohwy, H.J. Jakobsen, M. Eden, M.H. Levitt, N.C. Nielsen, J. Chem. Phys. 108 (1998) 2686-2694.

[10] M. Hohwy, C.M. Rienstra, C.P. Jaroniec, R.G. Griffin, J. Chem. Phys. 110 (1999) 7983-7992.

[11] A. Brinkmann, M. Eden, M.H. Levitt, J. Chem. Phys. 112 (2000) 8539-8554.

[12] J. Gunne, H. Eckert, Chem.-Eur. J. 4 (1998) 1762-1767.

[13] T. Gullion, S. Vega, Chem. Phys. Lett. 194 (1992) 423-428.

[14] A.E. Bennett, C.M. Rienstra, J.M. Griffiths, W.G. Zhen, P.T. Lansbury, R.G. Griffin, J. Chem. Phys. 108 (1998) 9463-9479.

[15] F.J. Blanco, R. Tycko, J. Magn. Reson. 149 (2001) 131-138.

[16] Y. Ishii, J. Chem. Phys. 114 (2001) 8473-8483.

[17] Y. Ishii, J.J. Balbach, R. Tycko, Chem. Phys. 266 (2001) 231-236.

[18] N.A. Oyler, R. Tycko, J. Phys. Chem. B 106 (2002) 8382-8389.

[19] Y.H. Tseng, J.H. Zhan, K.S.K. Lin, C.Y. Mou, J.C.C. Chan, Solid State Nucl. Magn. Reson. 26 (2004) 99-104.

[20] M. Bak, J.T. Rasmussen, N.C. Nielsen, J. Magn. Reson. 147 (2000) 296-330.

[21] M. Bak, N.C. Nielsen, J. Magn. Reson. 125 (1997) 132-139.

[22] M. Mathew, W.E. Brown, L.W. Schroeder, B. Dickens, J. Crystallogr. Spectrosc. Res. 18 (1988) 235-250.

[23] M.I. Kay, R.A. Young, A.S. Posner, Nature 204 (1964) $1050-1052$.

[24] Y. Wu, J.L. Ackerman, E.S. Strawich, C. Rey, H.M. Kim, M.J. Glimcher, Calcif. Tissue Int. 72 (2003) 610-626.

[25] T. Gullion, D.B. Baker, M.S. Conradi, J. Magn. Reson. 89 (1990) 479-484.

[26] R.R. Ernst, G. Bodenhausen, A. Wokaun, Principles of Nuclear Magnetic Resonance in One and Two Dimensions, Clarendon Press, Oxford, 1987.

[27] L.-S. Du, M.H. Levitt, C.P. Grey, J. Magn. Reson. 140 (1999) 242-249.

[28] J. Gunne, J. Magn. Reson. 165 (2003) 18-32. 